\title{
Perspective
}

\section{Red Blood Cell Distribution Width: Another Prognostic Factor for COVID-19?}

\author{
Maria Lagadinou $^{1, *}$, Despoina Gkentzi ${ }^{2}$ (D), Markos N. Marangos ${ }^{1}$, Fotini Paliogianni ${ }^{3}$, Elena E. Solomou ${ }^{1}$ \\ ${ }^{1}$ Department of Internal Medicine, University Hospital of Patras, Greece \\ ${ }^{2}$ Department of Pediatrics, University Hospital of Patras, Greece \\ ${ }^{3}$ Microbiology Department, University Hospital of Patras, Greece
}

\section{ARTICLE INFO}

\section{Article History}

Received 26 February 2021

Accepted 18 March 2021

\section{Keywords}

COVID-19

red blood cell distribution width

prognostic factor

\begin{abstract}
The coronavirus disease 2019 (COVID-19) is a pandemic with a high rate of hospitalization, admission to intensive care units, and mortality. Identifying patients at the highest risk for severe disease is important to facilitate early, aggressive intervention. High red blood cell distribution width (RDW) values are associated with increased mortality in the general population in patients suffering from several conditions, including cardiovascular disease, sepsis, acute kidney injury, chronic obstructive pulmonary disease, and hepatitis B. Our study aimed to determine whether RDW levels in all COVID-19 confirmed cases admitted to the Patras University Hospital, Greece, was an independent prognostic factor of hospitalization and disease outcome.
\end{abstract}

(C) 2021 International Academy for Clinical Hematology. Publishing services by Atlantis Press International B.V. This is an open access article distributed under the CC BY-NC 4.0 license (http://creativecommons.org/licenses/by-nc/4.0/).

\section{INTRODUCTION}

Coronavirus disease 2019 (COVID-19) is an acute respiratory illness which spread worldwide and is now a pandemic. This novel infection caused by the severe acute respiratory syndrome coronavirus 2 (SARS-CoV-2) presents with heterogenous clinical symptoms, with fever, dry cough, fatigue and dyspnoea being the most common, but can also lead to acute respiratory distress syndrome, and death [1]. An asymptomatic course of the disease has also been reported, mainly in young adults. Nevertheless, the disease evolution and potential predicting factors of mortality in all patients diagnosed with COVID-19 infection are poorly understood [2].

Coronavirus disease 2019 has a high rate of hospitalization, admission in intensive care units, and mortality. Identifying patients at the highest risk for severe disease is important to facilitate early, aggressive intervention, and to manage local hospital resources to reduce the crisis that has occurred in many hospital systems [3].

Red cell distribution width (RDW) is analyzed routinely in a complete blood count. It shows the heterogeneity in the red blood cell size and has a significant role in the differential diagnosis of anemia [4]. High RDW values are associated with increased mortality in patients suffering from cardiovascular disease, sepsis, acute kidney injury, chronic obstructive pulmonary disease, hepatitis B, and those on chronic dialysis [5]. Our study aimed to determine whether RDW levels upon admission of COVID-19 confirmed cases was an independent prognostic factor of hospitalization and disease outcome.

Corresponding author. Email: m_lagad2004@yahoo.gr

Peer review under responsibility of the International Academy for Clinical Hematology

\section{DATA COLLECTION}

Demographic characteristics and laboratory findings were collected and analyzed for all patients enrolled in this study after written informed consent. These were patients diagnosed with SARSCoV2 infection and referred to the Patras University Hospital.

Parameters from peripheral blood counts included white blood cells, RDW, lactate dehydrogenase ( $\mathrm{LDH})$, creatinine kinase, and coagulation parameters (fibrinogen, D-dimers). The need for hospitalization as well as the final outcome of the patients was also included in the analysis. Patients with a past medical history of thalassemia or thalassemia trait were excluded from the study.

\section{STATISTICAL ANALYSIS}

Statistical analysis of data was performed using SPSS-25 statistical software (IBM statistics SPSS version 25). Statistical values were expressed as mean $\pm \mathrm{SD}$. The minimum level of statistical significance, the $p$-value, was set as 0.05 . The Pearson correlation index was used for studying the relationship between variables, and the Mann-Whitney test was used to investigate the differences of variables between different and independent group populations.

\section{RESULTS}

This was a retrospective, one-center study. A total of 101 patients with laboratory-confirmed SARS-CoV-2 infection were enrolled. All patients were examined at the Emergency Department of the Patras University Hospital. 
The median age was $59.63 \pm 16.2$ years; $54 \%$ were male and $46 \%$ were female; $47 \%$ were elderly (>65 years old) and 53\% were younger than 65 years. Most patients presented with dry cough and high fever upon admission. Co-morbidities were present in 39 patients (38.6\%). Hematological and solid organ malignancies were the most common (30\%).

The RDW ranged from $11 \%$ to $18.8 \%$, with a median of $13.95 \pm 1.35 \%$. There was no significant difference in RDW between patients who were admitted $(n=79)$ and those who were treated as outpatients $(n=22)(14.04 \pm 1.34$ vs $13.33 \pm 0.82$ respectively, $p=0.132)$. Measurements are shown in Figure 1. However, older patients $(n=44)$ had a significantly higher RDW than individuals $<65$ years old $(n=57)(14.34 \pm 1.45$ vs $13.44 \pm 1.01$ respectively, $p=0.005)$, regardless of hospitalization.

Thirty-seven percentage of the subjects were the younger patients $(<65$ years old) who were admitted to the hospital. Twenty patients of the same age group were treated in an outpatient setting, representing $19.8 \%$ of all patients examined and enrolled in the study. The RDW value of the former group was significantly higher than in the latter ( 13 vs $13.48 \pm 1.05$ respectively, $p=0.031$ ). When analyzed with regards to their outcome, hospitalized younger patients who died had a significantly higher RDW than those of the same age group who recovered and were discharged $(15 \pm 1.41$ vs $13.25 \pm 0.85, p=0.04)$.

The same difference between patients who died and those who were discharged $(14.7 \pm 2.0$ vs $13.75 \pm 1.18$, respectively, $p=0.05)$ was observed in the whole group, regardless of their age. Measurements are shown in Figure 2. Among those patients who died, $80 \%(n=12)$ were older and 20\% $(n=3)$ were younger than 65 years. The mean RDW value among younger patients who died was $14.33 \pm 1.5$, which was not significantly different from that $(14.88 \pm 2.2)$ of older patients who died.

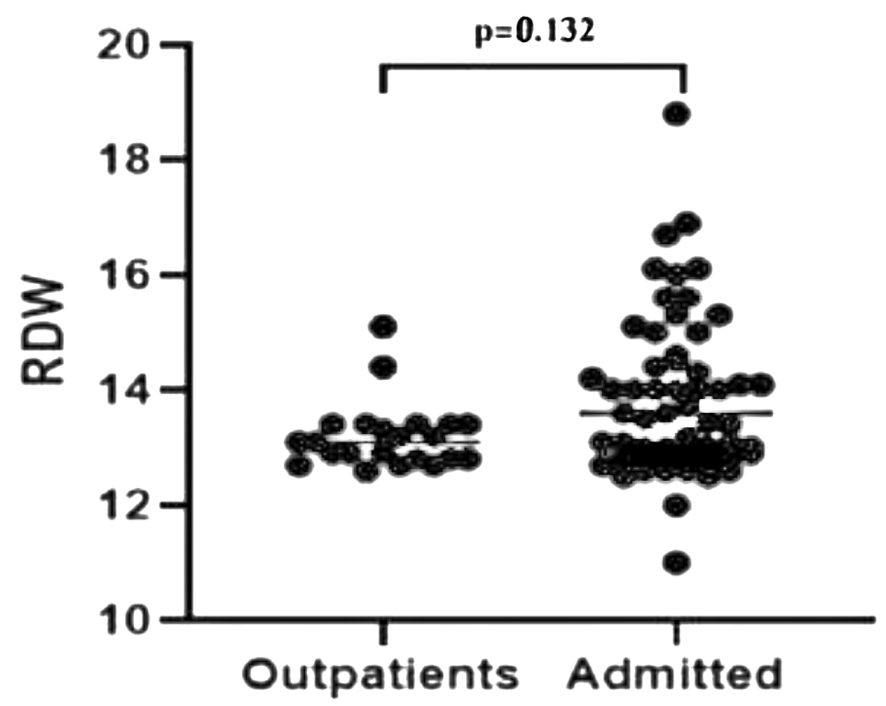

Figure 1 Measurements of RDW values according to hospital admission. The dot plots show the data from all the patients analyzed. Outpatients $(n=22)$ : represent the patients who were treated in an outpatient setting. Admitted $(n=79)$ : represent all patients who were admitted.
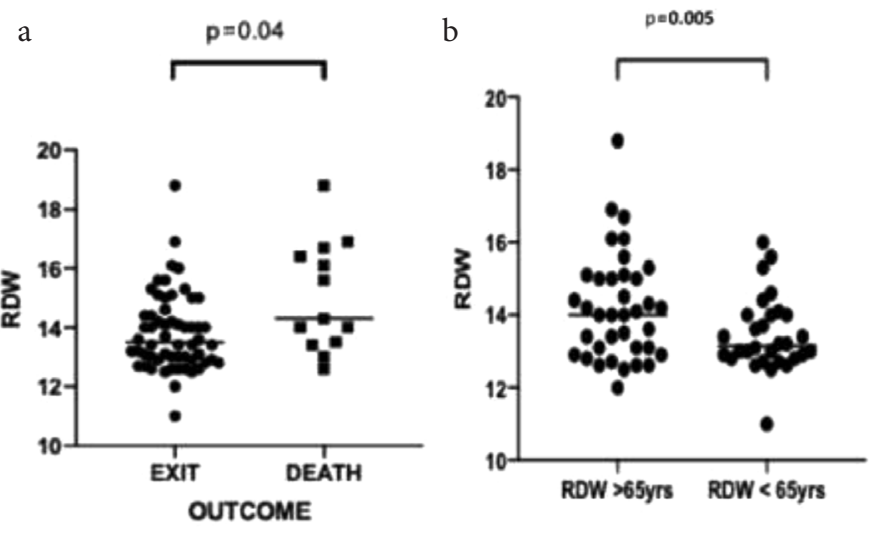

Figure 2 (a) The RDW represented according to outcome (recovery vs death) and (b) age ( $>65$ years vs $<65$ years). The dot plots show the data from all the patients analyzed.

There was no difference in the RDW values before and 1 week after hospitalization for all patients studied (analysis on all ages, $14.18 \pm 1.38$ vs $14.03 \pm 1.38$, respectively, $p=0.361$ ).

The RDW was associated with other laboratory parameters which are considered as prognostic factors for COVID-19 [6]. Specifically, elevated RDW was significantly correlated with increased D-dimers $(p=0.03)$. There were correlation trends between ferritin, $\mathrm{LDH}$, fibrinogen and RDW upon admission, but none of these was significant ( $p=0.098,0.662$ and 0.362 , respectively).

\section{DISCUSSION}

The COVID-19 pandemic caused by SARS-CoV-2 has led to numerous cases and deaths worldwide. It is a fact that the virus is spreading far more quickly and is very contagious [1,7].

Recently, a study has shown that RDW is associated with multiple inflammatory reactions and can predict the severity and prognosis of many diseases [8]. In this study, we found that RDW was significantly elevated in COVID-19 patients who eventually died. Our results are consistent with those from Foy et al. [3], who also found that patients whose RDW was increased during admission had an increased mortality risk. The RDW is a standard parameter of a routine complete blood count test and is, thus, easily available in any setting [3]. Although RDW is a nonspecific marker of illness, it can be useful in providing risk stratification in COVID-19 patients.

It is considered that high RDW is caused by conditions that lead to changes in erythropoiesis such as chronic inflammation, poor nutritional status, oxidative stress, and age-related diseases [5]. It is generally associated with an increased risk of mortality in various pathologic conditions (cancer, heart disease, pulmonary disease, sepsis, influenza, and cancer) [9]. The mechanism underlying this phenomenon is not completely understood.

In our study, we found that the RDW differed between patients who died and those who recovered. This finding would imply that routine blood tests may be additional useful tools for improving early prognosis and provide more intensive treatment [7]. 
In conclusion, the increased RDW as determined upon admission was associated with increased mortality. Although this is a retrospective, single-center study, RDW can be useful in categorizing patients who might benefit from early aggressive intervention.

\section{CONFLICTS OF INTEREST}

The authors declare they have no conflicts of interest.

\section{AUTHORS' CONTRIBUTION}

ML treated the patients, and wrote the manuscript. DG wrote the manuscript. MM and FP did the final approval. ES wrote and corrected the manuscript.

\section{REFERENCES}

[1] Mohanty SK, Satapathy A, Naidu MM, Mukhopadhyay S, Sharma S, Barton LM, et al. Severe acute respiratory syndrome coronavirus-2 (SARS-CoV-2) and coronavirus disease 19 (COVID-19) - anatomic pathology perspective on current knowledge. Diagn Pathol 2020;15;103.

[2] Perrotta F, Corbi G, Mazzeo G, Boccia M, Aronne L, D’Agnano V, et al. Correction to: COVID-19 and the elderly patients: insights into pathogenesis and clinical decision-making. Aging Clin Exp Res 2020;32;1909.

[3] Foy BH, Carlson JCT, Reinertsen E, Padros I Valls R, Pallares Lopez R, Palanques-Tost E, et al. Association of red blood cell distribution width with mortality risk in hospitalized adults with SARS-CoV-2 infection. JAMA Netw Open 2020;3; e2022058.

[4] Li N, Zhou H, Tang Q. Red blood cell distribution width: a novel predictive indicator for cardiovascular and cerebrovascular diseases. Dis Markers 2017;2017;7089493.

[5] Periša V, Zibar L, Sinčić-Petričević J, Knezović A, Periša I, Barbić J. Red blood cell distribution width as a simple negative prognostic factor in patients with diffuse large B-cell lymphoma: a retrospective study. Croat Med J 2015;56;334-43.

[6] Izcovich A, Ragusa MA, Tortosa F, Lavena Marzio MA, Agnoletti C, Bengolea A, et al. Prognostic factors for severity and mortality in patients infected with COVID-19: a systematic review. PLoS One 2020;15;e0241955.

[7] Lagadinou M, Salomou EE, Zareifopoulos N, Marangos M, Gogos C, Velissaris D. Prognosis of COVID-19: changes in laboratory parameters. Infez Med 2020;28;89-95.

[8] Zhang FX, Li ZL, Zhang ZD, Ma XC. Prognostic value of red blood cell distribution width for severe acute pancreatitis. World J Gastroenterol 2019;25;4739-48.

[9] Ellingsen TS, Lappegård J, Skjelbakken T, Braekkan SK, Hansen JB. Impact of red cell distribution width on future risk of cancer and all-cause mortality among cancer patients - the Tromsø Study. Haematologica 2015;100;e387-e9. 